\title{
CRYOPRESERVATION OF SHOOT-TIPS FROM DIFFERENT SUGARCANE VARIETIES USING D CRYO-PLATE TECHNIQUE
}

\author{
Tariq Rafique ${ }^{1,2}$, Shin-ichi Yamamoto, ${ }^{1, *}$ Kuniaki Fukui ${ }^{1}$, Daisuke Tanaka ${ }^{1}$, Miriam Valle \\ Arizaga $^{4}$, Mazhar Abbas ${ }^{3}$, Toshikazu Matsumoto ${ }^{5}$ and Takao Niino ${ }^{4}$ \\ ${ }^{1}$ Genebank, National Institute of Agrobiological Sciences (NIAS), 2-1-2 Kannondai, Tsukuba 305-8602, Japan; ${ }^{2}$ Plant \\ Genetic Resource Institute, National Agricultural Research Center, Islamabad 45500, Pakistan; ${ }^{3}$ Institute of \\ Horticultural Sciences, University of Agriculture, Faisalabad, Pakistan; ${ }^{4}$ University of Tsukuba, 1-1-1 Tennodai, \\ Tsukuba 305-8572, Japan; ${ }^{5}$ Shimane University, Matsue 690-1102, Japan. \\ "Corresponding author's e-mail: shiyam@affrc.go.jp
}

\begin{abstract}
Cryopreservation is an effective approach to conserve sugarcane germplasm for long term. This study was conducted to develop D cryo-plate technique to conserve sugarcane's shoot tips. This new and effective cryopreservation technique will serve the purpose to conserve sugarcane germplasm efficiently on the large scale at genebanks. Sugarcane variety Ni-1 were used to optimize the protocol and after optimization 11 other varieties were tested with optimized protocol. It was observed that shoot tips of Ni-1 with a length of 1.5 to $2.0 \mathrm{~mm}$, precultured on semi-solid 1/2 MS medium for 1 day and semi-solid MS medium with $0.3 \mathrm{M}$ sucrose for 1 day, treated with loading solution containing $2.0 \mathrm{M}$ glycerol $+1.2 \mathrm{M}$ sucrose for 30 min and air dehydrated in an air laminar flow's air current for $45 \mathrm{~min}$, removed from alginate gel after cryopreservation and kept in dark for seven days produced maximum regrowth (97.7\%). Range of $20.0 \%$ to $100 \%$ with an average of $52.1 \%$ was observed among sugarcane varieties for regrowth after cryopreservation with optimized D cryoplate protocol.

Keywords: Air dehydration, droplet vitrification, encapsulation dehydration, Sacharum officinarum, V cryo-plate
\end{abstract}

\section{INTRODUCTION}

The Food and Agriculture Organization of the United Nations estimates that sugarcane is the world's largest crop by production quantity. Approximately, it is grown in 90 countries and 1.83 billion tons is total production across the world. Sugarcane is not only important for its sugar content, which accounts for $80 \%$ of global sugar production, but also for many bi-products used in different industries (Anonymous, 2015). Sugarcane is a cross pollinated crop but is a very shy seed producer and it is not practical to store sugarcane seeds, even though they are orthodox seeds (Roberts, 1973). Sugarcane is mainly conserved in field collections where propagation uses cuttings and accessions need replanting every 1 to 5 years. In Japan, 1975 accessions including 592 wild accessions are maintained in field collections at different locations of the country. There is always the risk of adverse weather, pests or diseases destroying accessions in the field. In vitro conservation is an alternative to field collections. Sugarcane germplasm conserved by in vitro slow growth at CIRAD-CA Montpellier presently includes 650 sugarcane varieties (Paulet et al., 1991). Maintaining a huge in vitro collection is laborious because after a certain time reculturing is required to maintain the germplasm (Withers, 1987) and there is also the risk of contamination. The main concerns during germplasm conservation is the genetic stability, for in vitro conservation there is always a chance of somaclonal variation when sugarcane germplasm has been conserved by successive tissue culture.

Cryopreservation $\left(-196^{\circ} \mathrm{C}\right)$ of sugarcane is safe and practical for long term storage of sugarcane without fear of genetic changes because at this temperature biological activities completely stopped (Engelmann, 2004). Other benefits are the safety of germplasm from biotic and abiotic hazards, limited maintenance, reduced space and low maintenance costs. In the case of sugarcane, cryopreservation protocols have been developed for various materials: apices of in vitro plantlets using the encapsulation-dehydration technique (GonzalezArnao et al., 1993; Paulet et al., 1993); cell suspensions (Finkle and Ulrich, 1979) and embryogenic callus using classical freezing protocols (Eksomtramage et al., 1992; Gnanapragasam and Vasil, 1992; Jian et al., 1987) and simplified cryopreservation protocols (Martinez-Montero et al., 1998). Moreover, the cryopreservation procedure based on vitrification techniques for somatic embryos is published (Martinez-Montero et al., 2008).

Currently, effective V and D cryo-plate methods have been developed for many plant species (Vujovic et al., 2015). These protocols make the handling of shoot tips easy during the procedure and also have increased cooling and warming rate of shoot tips hence are effective and convenient for cryopreservation of various crops. In our former work, the $\mathrm{V}$ cryo-plate method has been successfully applied to 11 sugarcane varieties with an average regrowth rate of $70.3 \%$ 
(Rafique et al., 2015). The D cryo-plate method was proposed by Niino et al. (2013) using in vitro mat rush. This method could be used with large explants, which are very sensitive to physical damage and cryoprotectant toxicity (Niino et al., 2014).

The objectives of this study are to determine the applicability and optimization of the D cryo-plate method for use with sugarcane germplasm.

\section{MATERIALS AND METHODS}

Plant materials and tissue culture protocols: Sugarcane germplasm was acquired from sub-genebanks of Japan. Sugarcane variety $\mathrm{Ni}-1$ was used to optimize the conditions of the successive steps of the D cryo-plate method and also used in the droplet vitrification and encapsulation dehydration procedures. After optimization of the protocol, 11 additional varieties were also tested using the cryopreservation protocol developed with variety $\mathrm{Ni}-1$. Lateral buds from fully grown sugarcanes were excised, washed with "Muse" (Proctor and Gamble, Japan) medicated LN soap and surface-sterilized with $70 \%$ ethanol for $1 \mathrm{~min}$ and sodium hypochlorite solution with $2 \%$ active chlorine for $15 \mathrm{~min}$. After that buds were washed thrice with sterile water and from these sterilized buds, 2-4 mm shoot tips were excised using a stereomicroscope. Excised shoot tips were placed on $1 / 2 \mathrm{MS}$ semi-solid medium (Murashige and Skoog, 1962) containing 3\% sucrose, 0.01 $\mathrm{mg} / \mathrm{l}$ naphthalene acetic acid (NAA) and $0.1 \mathrm{mg} / \mathrm{l}$ benzyladenine (BA) in test tubes (Gonzalez-Arnao and Engelmann, 2006). Initially all the cultures were kept in the dark for 7 days and after that incubated in light with a photoperiod of $16 \mathrm{~h}$ light $\left(52 \mu \mathrm{mol} / \mathrm{m}^{2} \mathrm{~s}\right.$, standard condition $)$. In vitro shootlets were then transferred to $1 / 2 \mathrm{MS}$ semi-solid medium containing 3\% sucrose and $1 \mathrm{ml} / 1$ Plant Preservative Mixture (PPM) (manufactured by Plant Cell Technology, Inc. US) in jars with the size of $80 \times 102 \mathrm{~mm}$ (Fig. 1A).

Preconditioning and preculture: After removal of dead leaves, in vitro grown shoots of sugarcane containing shoot tips were cut to a size of 5-7 mm (Fig. 1B) and plated on semisolid 1/2 MS medium having 3\% sucrose in Petri dishes for 1 week to obtain uniform material for shoot tips. The shoot tips used were 1.5-2.0 mm long with a basal part (Fig. 1C). The excised shoot tips were cultured on semi-solid 1/2 MS medium containing 3\% sucrose for 1 day and precultured on semi-solid MS medium containing $0.3 \mathrm{M}$ sucrose for 1 day at room temperature (RT). For the experiment studying the

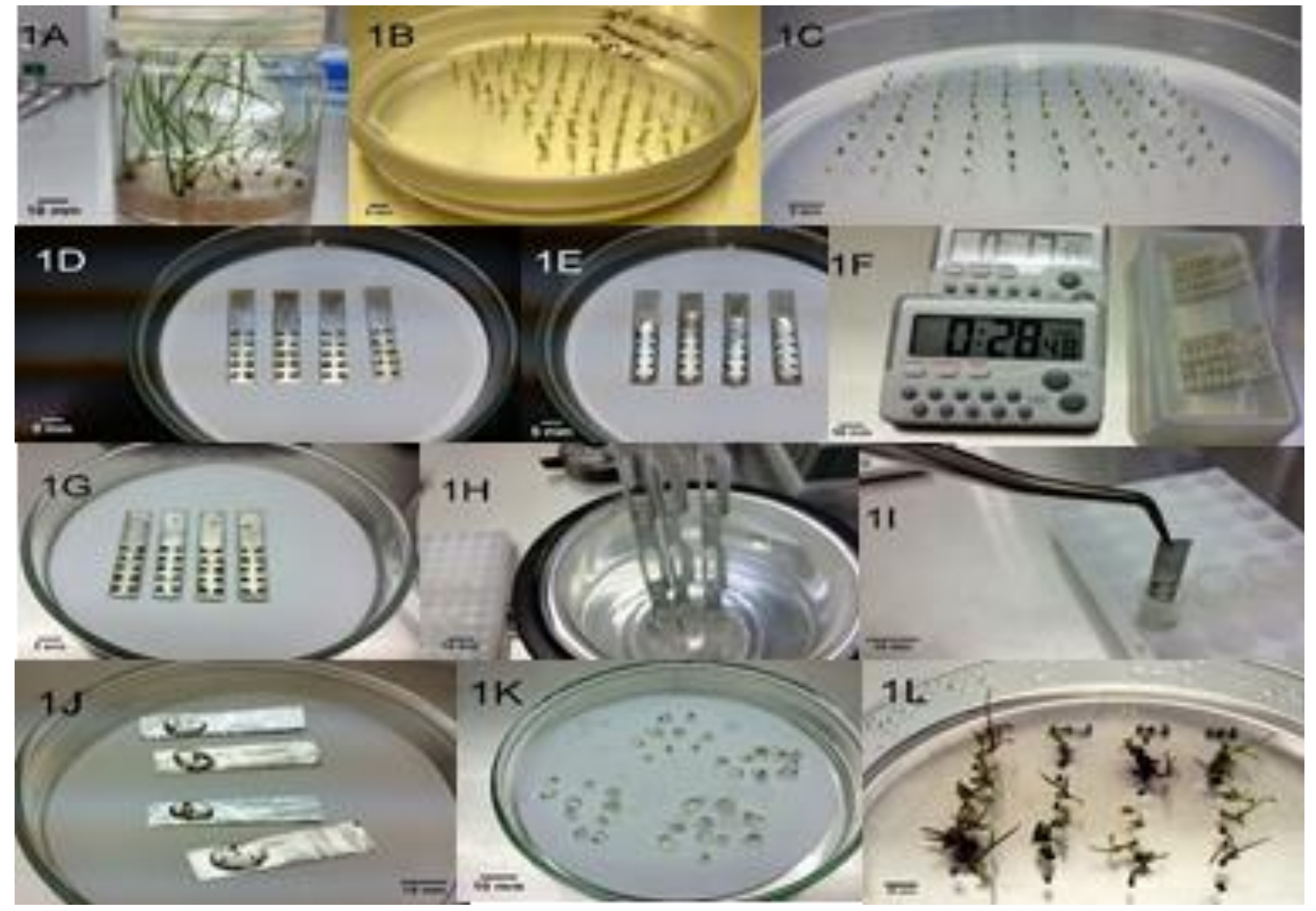

Figure 1. D cryo-plate procedure for sugarcane and regrowth after cryopreservation. 
effect of shoot tip size, three different sizes were used; 0.5$1.0,1.0-1.5$ and $1.5-2.0 \mathrm{~mm}$. For the preculture experiment, semi-solid MS medium containing $0.3 \mathrm{M}$ or $0.5 \mathrm{M}$ sucrose were used for 1 day preculture at RT.

D cryo-plate procedure: The steps of D cryo-plate procedure followed as. The * indicates the condition used in the standard procedure.

1. Pour sodium alginate solution in the wells of the aluminium cryo-plates using a micropipette at RT. The alginate solution contains $2 \%(\mathrm{w} / \mathrm{v})$ sodium alginate (viscosity 300-400 cps, Wako Pure Chem. Ind.) in calcium-free MS basal medium.

2. Place the precultured specimens (length $1.5-2.0 \mathrm{~mm}$, width $1.0-1.5 \mathrm{~mm}$ ) one by one in the wells (one bud/well) with the tip of a scalpel blade and slightly press the buds to make them fit in the wells; drip sodium alginate solution (about $1.0 \mu \mathrm{l}$ ) again on the buds using a micropipette (Fig. 1D).

3. Pour calcium chloride solution drop-wise (about 0.1 $\mathrm{ml} / \mathrm{plate}$ ) on the section of the aluminum plates where the buds are located until they are covered and wait for 15 min to achieve complete polymerization of sodium alginate (Fig. 1E). The calcium solution contains $0.1 \mathrm{M}$ calcium chloride in MS basal medium.

4. Remove the calcium chloride solution from the cryoplates by gently tapping the cryo-plates on filter paper.

5. Place the cryo-plates with the encapsulated specimens in a $25 \mathrm{ml}$ pipetting reservoir filled with about $20 \mathrm{ml}$ loading solution (LS) (Fig. 1F). The LS solution contains $2 \mathrm{M}$ glycerol $+0.8,1.2^{*}$ and $1.6 \mathrm{M}$ sucrose in liquid MS basal medium. The specimens are treated with $\mathrm{LS}$ at $25^{\circ} \mathrm{C}$ for $30 \mathrm{~min}$ to induce dehydration tolerance (osmoprotection).

6. Remove the cryo-plates from LS and place them in a Petri dish on filter paper after removing the excess LS solution. The specimens on the cryo-plates are desiccated under the air current of a laminar flow cabinet (HC-1600FS, Oriental Co. Japan) for 0, 15, 30, 45*, 60, 75, 90, 105 and 120 min at $25^{\circ} \mathrm{C}$, with $40-50 \%$ relative humidity (RH) (Fig. 1G).

7. After dehydration, transfer the cryo-plates in $2 \mathrm{ml}$ cryotubes, which are held on cryo-canes, and plunge them directly in liquid nitrogen (LN) where they are kept for at least $30 \mathrm{~min}$ (Figure 1H).

8. For rewarming, the cryotubes are retrieved from $L N$. The cryo-plates are immersed in cryotubes containing $2 \mathrm{ml}$ MS liquid medium with $1 \mathrm{M}$ sucrose (Fig. 1I), in which they are incubated for $15 \mathrm{~min}$ at RT.

9. Shoot tips were transferred to semi solid $1 / 2 \mathrm{MS}$ medium without growth regulators or with $0.01 \mathrm{mg} / \mathrm{l} \mathrm{NAA}+0.1$ $\mathrm{mg} / \mathrm{l} \mathrm{BA}$ * or with $0.01 \mathrm{mg} / \mathrm{l} \mathrm{NAA}+0.1 \mathrm{mg} / \mathrm{l} \mathrm{BA}+0.01$ $\mathrm{mg} / \mathrm{l} \mathrm{PVP}$ under standard conditions.

10. After transfer to regrowth medium, initially all the cultures were kept in the dark for 7 days and then transferred into light under standard conditions. In one experiment aiming at observing the effect of light exposure, the shoot tips were placed under light.

Measurement of shoot tips moisture content: Decrease in moisture content of sugarcane's shoot tips by air dehydration were calculated by weighing them after 15 min intervals of dehydration from 0 to 120 min. Dry weight was determined after drying the shoot tips for 48 hours at $102^{\circ} \mathrm{C}$ (Niino et al., 2014).

Droplet vitrification: Steps for droplet vitrification followed as.

1. Sugarcane shoot tips were placed in LS containing $1.2 \mathrm{M}$ sucrose for $30 \mathrm{~min}$.

2. Vitrification were carried out by plant vitrification solution number 2 (PVS2) which is composed of $0.4 \mathrm{M}$ sucrose, $150 \mathrm{~g} / \mathrm{l}$ glycol, $150 \mathrm{~g} / \mathrm{l}$ dimethyl sulfoxide and $300 \mathrm{~g} / \mathrm{l}$ glycerol, for $30 \mathrm{~min}$ at RT.

3. After that shoot tips were transferred to aluminium strips having $10 \mu 1$ drop of PVS2 (10 shoot tips per drop) (Fig. $1 \mathrm{~J}$ ) and directly plunged in to $\mathrm{LN}$ for $30 \mathrm{~min}$.

4. For rewarming of shoot tips, aluminum strips were transferred to $1.0 \mathrm{M}$ sucrose solution for $15 \mathrm{~min}$ at RT.

5. Shoot tips were then transferred to semi solid $1 / 2 \mathrm{MS}$ medium containing $0.01 \mathrm{mg} / \mathrm{l} \mathrm{NAA}+0.1 \mathrm{mg} / \mathrm{l} \mathrm{BA}$

6. For first 7 days after cryopreservation the cultures were held in the dark and then transferred to the light afterwards.

Encapsulation dehydration: 2\% sodium alginate were used to encapsulate shoot tips with a diameter of $4-5 \mathrm{~mm}$ for beads (Fig. 1K). Alginate beads containing shoot tips were kept in LS, for $30 \mathrm{~min}$, comprising of $1.2 \mathrm{M}$ sucrose. Afterwards alginate beads containing shoot tips were dehydrated in laminar flow's air current for $30 \mathrm{~min}$ and then submerged into $\mathrm{LN}$ for $30 \mathrm{~min}$. Subsequently the shoot tips were rewarmed in $1.0 \mathrm{M}$ sucrose solution at RT for $15 \mathrm{~min}$ and place on recovery medium as used for the D cryo-plate method. The cultures were retained in dark for 7 days and transferred to the light afterwards.

Data analysis: Regrowth (shoot elongation with or without root formation) was evaluated 4 weeks after rewarming of cryopreserved shoot tips. Each experimental treatment was replicated thrice with each replication containing 10 shoot tips. Statistical analysis was done by means of ANOVA and after that Tukey's honest significant difference (HSD) was used for means comparison. Significant differences were set at $\mathrm{P} \leq 0.05$. Means of replicates with standard error are presented in the Tables and Figures.

\section{RESULTS}

In the D cryo-plate procedure, preconditioning, preculture, osmoprotection with LS, dehydration with air and silica gel and post-rewarming handling of shoot tips are the key parameters for successful cryopreservation. 
Preconditioning and preculture: Shoot tip size significantly affects regrowth after cryopreservation, with increased shoot tip size success in regrowth improves (Table 1). Shoot tips having 1.5-2.0 $\mathrm{mm}$ size, displayed maximum (96.7\%) regrowth after cryopreservation. In order to prepare the shoot tips for cryopreservation and to make them suitable to obtain higher regrowth after cryo-storage, their preculture on medium having sucrose is an important step and when precultured on medium having $0.3 \mathrm{M}$ sucrose for 1 day at RT, regrowth was maximum (96.7\%) after cryopreservation, whereas regrowth was lower $(86.7 \%)$ in high sucrose concentration $(0.5 \mathrm{M})$ preculture medium (Table 2$)$.

Table 1. Effect of explant size on the regrowth of cryopreserved shoot tips using the $D$ cryo-plate method.

\begin{tabular}{ll}
\hline Explant size (mm) & Regrowth $(\% \pm$ SD) \\
\hline $0.5-1$ & $20.0 \pm 26.5 \mathrm{~b}$ \\
$1-1.5$ & $20.0 \pm 17.3 \mathrm{~b}$ \\
$1.5-2$ & $96.7 \pm 05.7 \mathrm{a}$ \\
\hline
\end{tabular}

Mean values followed by different letters differ significantly at the 0.05 probability level.

D cryo-plate procedure (osmoprotection and dehydration): After preculture of shoot tips on semi-solid MS medium with $0.3 \mathrm{M}$ sucrose, the effect of osmoprotection with LS containing

Table 2. Effect of sucrose concentration in preculture medium on cryopreserved shoot tips using the D cryo-plate method.

\begin{tabular}{ll}
\hline Sucrose concentration & Regrowth $(\% \pm$ SD) \\
\hline $0.3 \mathrm{M}$ sucrose, 1 day & $96.7 \pm 5.7 \mathrm{a}$ \\
$0.5 \mathrm{M}$ sucrose, 1 day & $86.7 \pm 5.7 \mathrm{~b}$ \\
\hline
\end{tabular}

Mean values followed by different letters differ significantly at the 0.05 probability level.

Different sucrose concentrations and duration of dehydration with air drying was evaluated (Table 3 ). The results suggest that maximum regrowth $(96.7 \%)$ after cryo-storage was obtain with LS having $2.0 \mathrm{M}$ glycerol $+1.2 \mathrm{M}$ sucrose and dehydrated under air laminar flow's air current for $45 \mathrm{~min}$. These condition were selected as the optimal for other sugarcane varieties.

The relation of moisture content of shoot tips with alginate gel and the regrowth after cryo-storage at 15 min intervals of drying from 0 to 120 min was estimated after LS treatment with $2 \mathrm{M}$ glycerol $+1.2 \mathrm{M}$ sucrose. The regrowth percentage compared with the moisture content in shoot tips (Fig. 2 and 3 ) indicated that 28 to $35 \%$ moisture content in shoot tips is optimum prior to cryo-storage.

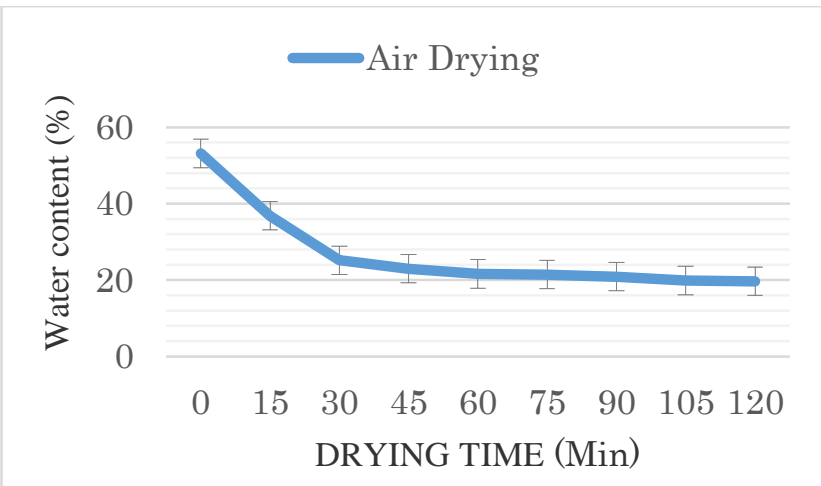

Figure 2. Decrease of moisture content of shoot tips with exposure to air drying.

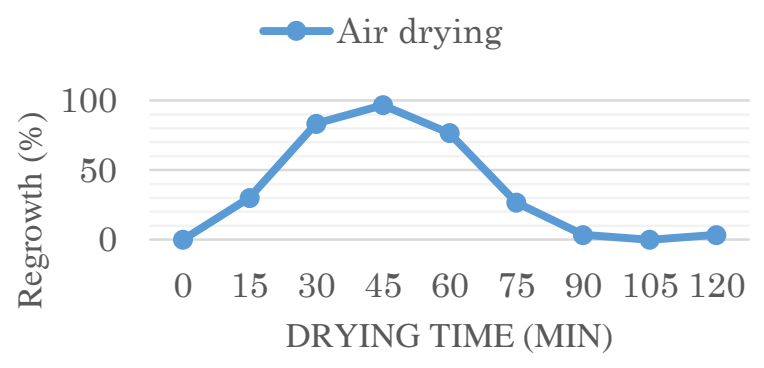

Figure 3. Regrowth curve of cryopreserved shoot tips with respect to air drying time.

Table 3. Effect of sucrose concentration in LS and drying time by air drying on the regrowth of cryopreserved shoot tips using the D cryo-plate method.

\begin{tabular}{lll}
\hline Sucrose concentration in LS & Drying Time & Regrowth rate (\% +SD) \\
\hline LS 0.8 & $30 \mathrm{~min}$ & $83.3 \pm 15.3 \mathrm{a}$ \\
& $45 \mathrm{~min}$ & $83.3 \pm 5.8 \mathrm{a}$ \\
LS 1.2 & $60 \mathrm{~min}$ & $70 \pm 10 \mathrm{abc}$ \\
& $30 \mathrm{~min}$ & $86.7 \pm 5.8 \mathrm{a}$ \\
LS 1.6 & $45 \mathrm{~min}$ & $96.7 \pm 5.8 \mathrm{a}$ \\
& $60 \mathrm{~min}$ & $76.7 \pm 11.5 \mathrm{ab}$ \\
& $30 \mathrm{~min}$ & $66.7 \pm 5.8 \mathrm{abcd}$ \\
& $45 \mathrm{~min}$ & $66.7 \pm 11.5 \mathrm{abcd}$ \\
\hline
\end{tabular}

Mean values followed by different letters differ significantly at the 0.05 probability level. 
Post-warming regrowth: Post-warming manipulations are important to obtain high regrowth after cryopreservation. Removing alginate gel from shoot tips significantly increased post-LN regrowth compared to shoot tips covered with alginate gel (Table 4). The initial light conditions for cryopreserved shoot tips after rewarming affected regrowth significantly as if placed in standard light conditions, their regrowth was significantly lower compared to shoot tips which were held in dark for 7 days (Table 5). The hormonal composition in the culture media also affected regrowth after cryopreservation. The addition of $0.01 \mathrm{mg} / \mathrm{l} \mathrm{NAA}$ and 0.1 $\mathrm{mg} / \mathrm{l} \mathrm{BA}$ in the culture medium produced maximum regrowth (100\%), whereas regrowth was only $80 \%$ on medium having $0.01 \mathrm{mg} / \mathrm{l} \mathrm{NAA}+0.1 \mathrm{mg} / \mathrm{l} \mathrm{BA}+0.01 \mathrm{mg} / \mathrm{l} \mathrm{PVP}$ and regrowth of $40 \%$ was observed on medium devoid of growth regulators (Table 6).

Table 4. Effect of alginate beads presence or absence on cryopreserved shoot tips using the D cryo-plate method.

\begin{tabular}{ll}
\hline Ag beads & Regrowth $(\% \pm$ SD) \\
\hline Present & $40.0 \pm 10 \mathrm{~b}$ \\
Removed & $96.7 \pm 5.7 \mathrm{a}$
\end{tabular}

$\mathrm{M}_{\text {ean values followed by different letters differ significantly }}$ at the 0.05 probability level.

Table 5. Effect of light on the regrowth of cryopreserved shoot tips using the D cryo-plate method.

\begin{tabular}{ll}
\hline Regrowth condition & Regrowth $(\% \pm$ SD $)$ \\
\hline In dark for 7 days & $96.7 \pm 5.7 \mathrm{a}$ \\
Without dark & $36.7 \pm 5.7 \mathrm{~b}$ \\
\hline
\end{tabular}

Mean values followed by different letters differ significantly at the 0.05 probability level

Optimal D cryo-plate procedure: The optimal conditions established for Ni-1 were as follows: 1.5 to $2.0 \mathrm{~mm}$ long shoot tips were placed on semi-solid 1/2 MS medium having 3\% sucrose for 1 day, 1 day preculture on semi-solid MS medium containing $0.3 \mathrm{M}$ sucrose, $30 \mathrm{~min}$ Osmoprotection by LS having $2.0 \mathrm{M}$ glycerol $+1.2 \mathrm{M}$ sucrose at $25^{\circ} \mathrm{C}$ and air dehydrated in air flow laminar hood for $45 \mathrm{~min}$ at RT before direct dipping in LN. For regeneration, cryopreserved shoot tips were rewarmed in $1.0 \mathrm{M}$ sucrose solution for $15 \mathrm{~min}$ at RT and plated on semi-solid 1/2 MS medium containing 3\% sucrose, $0.01 \mathrm{mg} / \mathrm{l} \mathrm{NAA}$ and $0.1 \mathrm{mg} / \mathrm{l} \mathrm{BA}$ after removal of the alginate gel. Cultures were retained in the dark for 7 days and shifted in standard light afterwards.

These optimal conditions were applied to an additional 11 varieties of sugarcane. Regrowth ranged from 20 to $100 \%$, with an average of $52.1 \%$ for the twelve varieties tested (Table 7).

Encapsulation dehydration and droplet vitrification: The regrowth percentage of sugarcane shoot tips after cryopreservation using the encapsulation dehydration and droplet vitrification methods were 13.3 and $30 \%$, respectively and significantly lower than the D cryo-plate method (Table 8).

A) In vitro plantlet of sugarcane in jar; B) Preconditioning of shoots on 1/2 MS medium to get uniform shoot tips;

C) Preculture on semi-solid MS medium with $0.3 \mathrm{M}$ sucrose; D) Shoot tips mounted on cryo-plate with sodium alginate solution; E) Hardening of gel with calcium solution; F) Shoot tip treatment with LS; G) Air drying in laminar flow; H) Immersion of cryotubes containing cryo-plates in LN; I) Warming in 1.0 M sucrose solution after LN exposure; J) Shoot tips on aluminum foil strips in $10 \mathrm{~m}$ PVS2 soln. for droplet vitrification; K) Encapsulation of shoot tips in alginate beads for encapsulation dehydration; L) Regenerated shoot tips after LN exposure (4 weeks after rewarming).

Table 8. Comparison of the regrowth percentage of sugarcane varieties after cryopreservation with optimum D cryo-plate method, encapsulation dehydration and droplet vitrification.

\begin{tabular}{ll}
\hline Methods & Regrowth $(\% \pm$ SD) \\
\hline D cryoplate & $96.7 \pm 5.7 \mathrm{a}$ \\
Encapsulation dehydration & $13.3+11.5 \mathrm{~b}$ \\
Droplet vitrification & $30.0+10.0 \mathrm{~b}$ \\
\hline
\end{tabular}

Table 6. Effect of recovery medium on the regrowth of cryopreserved shoot tips using the D cryo-plate method.

\begin{tabular}{lc}
\hline Medium & Regrowth (\% $\%$ SD) \\
\hline $1 / 2 \mathrm{MS}+30 \mathrm{~g} / \mathrm{l}$ sucrose & $40.0 \pm 20 \mathrm{~b}$ \\
$1 / 2 \mathrm{MS}+30 \mathrm{~g} / \mathrm{l}$ sucrose + NAA+ BA & $96.7 \pm 5.7 \mathrm{a}$ \\
$1 / 2 \mathrm{MS}+30 \mathrm{~g} / \mathrm{l}$ sucrose + NAA + BA + PVP & $80.0 \pm 10 \mathrm{a}$ \\
\hline
\end{tabular}

Mean values followed by different letters differ significantly at the 0.05 probability level

Table 7. Regrowth percentage of sugarcane varieties after cryopreservation with optimum D cryo-plate conditions.

\begin{tabular}{|c|c|c|c|c|c|c|c|c|c|c|c|c|}
\hline $\begin{array}{l}\text { S. No } \\
\text { Varieties }\end{array}$ & $\begin{array}{c}1 \\
\text { Chunnee }\end{array}$ & $\begin{array}{c}2 \\
\mathrm{NiF} 4\end{array}$ & $\begin{array}{c}3 \\
\text { NiF3 }\end{array}$ & $\begin{array}{c}4 \\
\mathrm{Ni} 27 \\
\end{array}$ & $\begin{array}{c}5 \\
\text { Badilla }\end{array}$ & $\begin{array}{c}6 \\
\text { Ni12 } \\
\end{array}$ & $\begin{array}{c}7 \\
\text { KNOO-114 }\end{array}$ & $\begin{array}{c}8 \\
\text { Kouchi Zairai }\end{array}$ & $\begin{array}{c}9 \\
\text { Wakayama }\end{array}$ & $\begin{array}{c}10 \\
\mathrm{NiF} 8\end{array}$ & $\begin{array}{c}11 \\
\mathrm{Ni}-1\end{array}$ & $\begin{array}{c}12 \\
\text { NiN2 }\end{array}$ \\
\hline $\begin{array}{l}\text { Regrowth } \\
\pm \text { SD }\end{array}$ & $\begin{array}{c}50.0 \\
\pm 17.3\end{array}$ & $\begin{array}{l}100.0 \\
\pm 0.0\end{array}$ & $\begin{array}{c}47.0 \\
\pm 11.5\end{array}$ & $\begin{array}{l}77.0 \\
\pm 5.7\end{array}$ & $\begin{array}{c}60.0 \\
\pm 10.0\end{array}$ & $\begin{array}{c}50.0 \\
\pm 10.0\end{array}$ & $\begin{array}{c}20.0 \\
\pm 10.0\end{array}$ & $\begin{array}{c}27.0 \\
\pm 11.5\end{array}$ & $\begin{array}{l}20.0 \\
\pm 0.0\end{array}$ & $\begin{array}{l}57.0 \\
\pm 5.7\end{array}$ & $\begin{array}{l}96.7 \\
\pm 5.7\end{array}$ & $\begin{array}{c}20.0 \\
\pm 10.0\end{array}$ \\
\hline
\end{tabular}

Mean values followed by different letters differ significantly at the 0.05 probability level. 
Mean values followed by different letters differ significantly at the 0.05 probability level.

\section{DISCUSSION}

There are very few reports on sugarcane cryopreservation using in vitro grown shoot tips. Successful cryopreservation was accomplished by the encapsulation dehydration procedure (Gonzalez-Arnao, 1993; Paulet et al., 1993). Recently, successful cryopreservation in vitro shoot tips of two sugarcane varieties were carried out by encapsulationdehydration and droplet vitrification with recovery 53-60\% and 20-37\%, respectively (Barraco et al., 2011). Using 11 sugarcane varieties regrowth rates of 56.7 to $100 \%$, with an average of $70.3 \%$, was achieved using an optimally developed V cryo-plate procedure (Rafique et al., 2015).

It is important to have different protocols for cryopreservation, because there are many types of plant propagules and plant species/varieties to be cryopreserved (Niino and Arizaga, 2015). Also, the protocols might efficiently complement one another (Niino et al., 2014). Despite research conducted to date, some genotypes are considered 'recalcitrant materials'. Kaczmarczyk et al. (2011) emphasized that regrowth after cryopreservation in different genotypes of potato is varied greatly because of the regeneration capacity of genotypes. As some genotypes of potato does not have regeneration ability in vitro and it is essential to store them as tuber (Keller et al., 2011). Several cryopreservation protocols developed are useful to overcome the different responses of genotype. These genotypic response differences include sensitivity to biochemical and osmotic toxicity of plant vitrification solution, weakness to dehydration or sub-zero temperatures, damage to plant material during dissection and post regrowth. In this paper, we assessed the feasibility of sugarcane shoot tip cryopreservation using the $\mathrm{D}$ cryo-plate method after optimizing different conditions. Average regrowth rate of 12 sugarcane varieties ranged from 20 to $100 \%$, with an average of $52.1 \%$. This average regrowth rate is higher than most previous studies but lower than that reported for the V cryoplate procedure (Rafique et al., 2015). Comparing the regrowth rates of nine varieties by both procedures in detail, 4 varieties (Ni-1, Ni27, Badilla and Ni12) were similar, 4 varieties (Chunnee, NiF3, KN00-114 and NiN2) had higher regrowth rates with the $\mathrm{V}$ cryo-plate method, and 1 variety (NiF4) had a higher regrowth rates with the D cryo-plate method (Rafique et al., 2015). These results show that some but not all varieties of sugarcane respond better to the $\mathrm{V}$ cryoplate method. In a mat rush cryopreservation experiment, the regrowth using D cryo-plate method was much more successful than the $\mathrm{V}$ cryo-plate method and regrowth rate ranged from 73.3 to $96.7 \%$, with an average of $86.3 \%$, compared to $52.5 \%$ for the $\mathrm{V}$ cryo-plate method. The 11 lines which displayed regrowth less than $50 \%$ with the $\mathrm{V}$ cryo-plate method, showed regrowth between 73.3-93.3\% with the D cryo-plate method (Niino et al., 2013). In an experiment with potato germplasm, average regrowth rates of cryopreserved shoot tips in V cryo-plate and D cryo-plate methods were 98.6\% and 91.6, respectively (Yamamoto et al., 2014). In cherry plum and plum cultivars, regrowth rates shoot tips cryopreservation by $\mathrm{V}$ cryo-plate and $\mathrm{D}$ cryo-plate methods were $41.7 \%$ and $77.5 \%, 44.6 \%$ and $47.5 \%$, respectively (Vujovic et al., 2015). Recently, the D cryo-plate method has been adapted to several plant species, data palm (Salma et al., 2014), Chenopodium odorum (Engelmann-Sylvestre and Engelmann, 2015), persimmon (Matsumoto et al., 2015), blueberry (Dhungana et al., 2015) and taro (Fukui et al., 2015). These results indicate that the D cryo-plate procedure helps to overcome problems associated with sensitivity to vitrification solutions, mistiming dehydration and damage to plant material during dissection (Vujovic et al., 2015). In the D cryo-plate protocol, the duration of physical dehydration is crucial for obtaining high post-LN recovery. The dehydration of shoot tips are influenced by the size/volume of shoot tips, the structure of shoot tips, preculture and LS conditions, and desiccation method. Usually, simple and small shoot tips may be appropriate for the $\mathrm{V}$ cryo-plate method and complicated and large shoot tips may be suitable for the D cryo-plate method. To explain these results it can be hypothesized that smaller specimens with the buds (the meristematic dome) exposed are more rapidly and uniformly dehydrated by PVS2, compared to larger shoots with the buds (the meristematic dome) covered by leaf sheaths. By contrast, physical dehydration in the D cryo-plate might be more uniform, explaining the equivalent regrowth obtained with small and large specimens (Niino et al., 2013).

Major advantages of $\mathrm{D}$ cryo-plate technique can be summarized as: handling of samples throughout the procedure is simple and quick; the possibility of injuring and losing shoot tips is considerably reduced compared with other methods; larger specimens can be used; and the window of optimal moisture contents for cryopreservation is very broad. Depending on the species and variety both the D cryo-plate and $\mathrm{V}$ cryo-plate methods are efficient and practical for cryobanking. The D cryo-plate protocol described here appears promising for cryopreservation of, not only sugarcane, but also other plants after marginal modifications of the procedure.

Reed (2001) stated that the physiological condition of the stock plants from which explants are taken as well as growth conditions after rewarming have a major impact on cryopreservation results. Investigations of preconditioning, preculture, osmoprotective treatment, an efficient tissue culture procedure and post-rewarming handling system are necessary for achieving high recovery after $\mathrm{LN}$ exposure in cryopreservation by V cryo-plate procedure (Rafique et al., 2015). In this paper, the following factors affected regrowth using the D cryo-plate procedure: shoot tip size, sucrose 
concentration in preculture medium, sucrose concentration in the LS and drying time by air flow, regrowth medium, regrowth light condition and removal of alginate gel from shoot tips. It is necessary to test each plant species at each step of both the $\mathrm{V}$ and $\mathrm{D}$ cryo-plate procedure to develop the best cryo-banking protocol. After modifications, the procedure can be used for practical cryobanking. To realize comprehensive cryo-storage of plant genetic resources, further development of cryopreservation techniques, diligent attention to protocol, and also best procedures for establishment of in vitro shoot culture system are necessary.

Acknowledgements: We are grateful to Shigeki Nakayama for providing germplasm for the experiments and to Ms. Naoko Nohara, Ms. Akiko Nishiuchi, Ms.Noriko Ishikura and Ms. Yumi Sato for their technical assistance. This work was mainly supported by a grant from Ministry of Agriculture, Forestry, and Fisheries of Japan (Genomics-based Technology for Agricultural Improvement, CRS-1001).

\section{REFERENCES}

Anonymous. 2015. Crop data, Food and Agriculture Organization of the United Nations.

Barraco, G., I. Sylvestre and F. Engelmann. 2011. Comparing encapsulation-dehydration and droplet-vitrification for cryopreservation of sugarcane (Saccharum spp.) shoot tips. Sci. Hortic. 130: 320-324.

Dhungana, S.A., H. Kunitake, T. Niino, S. Yamamoto, K. Fukui and T. Matsumoto. 2015. Cryopreservation of blueberry (Vaccinium L.) shoot tips by D cryo-plate method. Hort. Res. (Japan) 14: 363.

Eksomtramage, T., F. Paulet, E. Guiderdoni, J.C. Glaszmann and F. Engelmann. 1992 Development of a cryopreservation process for embryogenic calluses of a commercial hybrid of sugarcane (Saccharum sp.) and application to different varieties. CryoLetters 13: 239-52.

Engelmann, F. 2004. Plant cryopreservation: progress and prospects. In Vitro Cell \& Dev. Biol.-Plant 40: 427-433.

Engelmann-Sylvestre, I. and F. Engelmann. 2015. Cryopreservation of in vitro-grown shoot tips of Clinopodium odorum using aluminum cryo-plates. In Vitro Cell. Dev. Biol.-Plant 51:185-191.

Finkle, B.J. and J.M. Ulrich. 1979. Effect of cryoprotectants in combination on the survival of frozen sugarcane cells. Plant Physiol. 63: 598-604.

Fukui, K., S. Yamamoto, T. Rafique, T. Matsumoto and T. Niino. 2015. Cryopreservation of taro shoot tips using aluminum cryo-plates. Hort. Res. (Japan) 14 supple. 2: 402.

Gnanapragasam, S. and I.K. Vasil. 1990. Plant regeneration from a cryopreserved embryogenic cell suspension of a commercial sugarcane hybrid (Saccharum sp.). Plant Cell Rep. 9: 419-423.
Gnanapragasam, S. and I.K. Vasil. 1992. Cryopreservation of immature embryos, embryogenic callus and cell suspension cultures of gramineous species. Plant Sci. 83: 205-215.

Gonzalez-Arnao, M.T. and F. Engelmann. 2006. Cryopreservation of plant germplasm using the encapsulation dehydration technique: review and case study on sugarcane. CryoLetters 27: 155-168.

Gonzalez-Arnao, M.T., F. Engelmann, C. Huet and C. Urra. 1993. Cryopreservation of encapsulated apices of sugarcane: effect of freezing procedures and histology. CryoLetters 14: 303-308.

Jian, L.C., D.L. Sun and L.H. Sun. 1987. Sugarcane callus cryopreservation. In: P.H. Li (ed.), Plant cold hardiness, pp.323-337. Alan R. Liss, Inc., New York. ISBN 0-8451$1084-8$.

Kaczmarczyk, A., V.M. Rokka and E.R.J. Keller. 2011. Potato shoot tip cryopreservation: A review. Potato Res. 54: 45-79.

Keller, E.R.J., A. Sunura, C.H. Zanke, M. Grube and A. Kaczmarczyk. 2011. Cryopreservation and in vitro culture- State of the art as conservation strategy for genebank. Acta Hort. 918: 99-111.

Martinez-Montero, M.E., M.T. Gonzalez-Arnao, C. BorrotoNordelo, C. Puentes-Diaz and F. Engelmann. 1998. Cryopreservation of sugarcane embryogenic callus using a simplified freezing process. CryoLetters 19: 171-176.

Martinez-Montero, M.E., J. Martinez and F. Engelmann. 2008. Cryopreservation of sugarcane somatic embryos. CryoLetters 29: 229-242.

Matsumoto, $_{2}$ T., S. Yamamoto, K. Fukui, T. Rafique, F. Engelmann and T. Niino. 2015. Cryopreservation of persimmon shoot tips from dormant buds using the D cryo-plate technique. The Hort. J. 84:106-110.

Murashige, T. and F. Skoog. 1962. A revised medium for rapid growth and bioassays with tobacco cultures. Physiol. Plant. 15:473-497.

Niino, T. and M.V. Arizaga. 2015. Cryopreservation for preservation of potato genetic resources. Breeding Sci. 65:41-52.

Niino, T., S. Yamamoto, K. Fukui, C.R.C. Martínez, M.V. Arizaga, T. Matsumoto and F. Engelmann. 2013. Dehydration improves cryopreservation of mat rush (Juncus decipiens Nakai) basal stem buds on cryo-plates. CryoLetters 34: 549-560.

Niino, T., Wunna, K. Watanabe, N. Nohara, T. Rafique, S. Yamamoto, K. Fukui, M.V. Arizaga, C.R.C. Martinez, T. Matsumoto and F. Engelmann. 2014 Cryopreservation of mat rush lateral buds by air dehydration using aluminum cryo-plate. Plant Biotech. 31:281-287.

Paulet, F., C. Acquaviva, T. Eksomtramage, Y.H. Lu, A. D'Hont and J.C. Glasmann. 1991. In Actes de la Première 
Rencontre Internationale Française sur la Canne à Sucre. AFCAS, Nogent-Sur-Mame, France, pp.47-52.

Paulet, F., F. Engelmann and J.C. Glaszmann. 1993. Cryopreservation of apices of in vitro plantlets of sugarcane (Saccharum sp. hybrids) using encapsulation/dehydration. Plant Cell Rep. 12: 525-529.

Rafique, T., S. Yamamoto, K. Fukui, Z. Mahmood and T. Niino. 2015. Cryopreservation of sugarcane using the $\mathrm{V}$ cryo-plate technique. CryoLetters 36:51-59.

Reed, B.M. 2001. Implementing cryogenic storage of clonally propagated plants. CryoLetters 22: 97-104.

Roberts, E.H. 1973. Predicting the storage life of seeds. Seed Sci. Technol. 1: 499-514.

Salma, M., L. Fki, I. Engelmann-Sylvestre, T. Niino and F. Engelmann. 2014. Comparison of droplet- vitrification and D-cryoplate for cryopreservation of date palm
(Phoenix dactylifera L.) polyembryonic masses. Sci. Hortic. 179: 91-97.

Ulrich, J.M., B.J. Finkle and P.H. Moore. 1984. Frozen preservation of cultured sugarcane cells. Sugarcane 3:1114.

Vujovic, T., P. Chatelet, D. Ruzic and F. Engelmann. 2015. Cryopreservation of Prunus spp. using aluminum cryoplates. Sci. Hortic. 195:173-182.

Withers, L.A. 1987. Long-term preservation of plant cells, tissues and organs. Oxf Surv Plant Molecular Cell Biol 4: 221-272.

Yamamoto, S., Wunna, T. Rafique, M.V. Arizaga, K. Fukui, E.C. Gutierrez, C.R.C. Martinez, K. Watanabe and T. Niino. 2015. The aluminum cryo-plate increases efficiency of cryopreservation protocols for potato shoot tips. Am. J. Potato Res. 92:250-257. 